\title{
Cubic B-spline collocation method for solving time fractional gas dynamics equation
}

\author{
A. Esen ${ }^{1}$ and O. Tasbozan ${ }^{2}$ \\ 1 Department of Mathematics, Faculty of Science and Art, Inönü University, Malatya, 44280, Turkey \\ 2 Department of Mathematics, Faculty of Science and Art, Mustafa Kemal University, Hatay, 31000, \\ Turkey \\ E-mail: orkun.tasbozan@inonu.edu.tr
}

\begin{abstract}
In the present manuscript, a cubic B-spline finite element collocation method has been used to obtain numerical solutions of the nonlinear time fractional gas dynamics equation. While the Caputo form is used for the time fractional derivative appearing in the equation, the $L 1$ discretization formula is applied to the equation in terms of time. It has been seen that the results of the present study are in agreement with the those of exact solution. Therefore, the present method can be used as an alternative and efficient one to find out the numerical solutions of both linear and nonlinear fractional differential equations available in the literature. In order to control the accuracy and efficiency of the present method, the error norms $L_{2}$ and $L_{\infty}$ have been calculated. It is evident that the newly obtained numerical solutions by the present method can be computed easily with the implementation and effectiveness of the approach used in the article.
\end{abstract}

2010 Mathematics Subject Classification. 97N40. 65N30, 65D07, 74S05

Keywords. Finite element method, collocation, time fractional gas dynamics equation, cubic B-spline.

\section{Introduction}

Fractional calculus has been an indispensable part of applied mathematics owing to its capasity in dealing with derivatives and integrals of non-integer orders. Its application to differentiation and integration having non-integer orders can be traced back to early in history, thus it is obvious that it is not new by no means. To be more precise, interest in the subject originates with almost with the ideas of the classical calculus [1]. But, especially over the last a few years, many authors have recognized that derivatives and integrals having non-integer order can be very suitable for the real description of many physical phenomena in the universe. Because, it has been repeatedly demonstrated that new fractional-order models are much better than previously used integer-order ones. Again, the ever growing number of fractional derivative applications in such diverse fields of science and engineering clearly shows the fact that there is a tremendous demand for a better mathematical modelings of real objects, and that the fractional calculus is one of the possible alternatives to provide more adequate mathematical modelings of real world objects and processes. In fact, among others, they have already been widely utilized for modelling problems encountered in such diverse fields as fluid mechanics, acoustics, biology, electromagnetism, diffusion, signal processing, and many other physical processes, one can look at $[2,3,4]$ and references therein. 
The finite element method plays an important role in solving both ordinary and partial differential equations. But, in the present paper, the method is going to be applied for solving time fractional nonlinear differential equation, namely time fractional gas dynamics equation. The main idea behind the finite element method is to discretize the entire region of the problem domain into an equivalent system of finite elements having associated nodes and to select the most suitable element type which will model most appropriately the real physical behavior. Therefore, by the finite element method, a complicated equation is turned into several solvable set of equations. The finite elements can be adjusted small enough to result in usable sizes and yet large enough to decrease the computational load $[5]$.

In the literature, one can find a wide range of studies dealing with obtaining analytical and numerical solutions of fractional differential equations with several different techniques and methods. Even though there exist few analytical methods such as found by Refs. $[6,7,8,9]$ providing exact solutions of the fractional equations, the numerical ones can be found more commonly and as the most appropriate and even sometimes the only alternative for dealing with most of the problems involving fractional equations. Because of this fact, it is very important to find and implement effective, accurate and easy numerical methods. Despite the fact that there still exist several methods applied for the solution of fractional partial differential equations, it is again a well known fact that there is still a long way to go in the area. There are several studies about fractional equations in the literature. Among others, Esen et. al. [10, 11] have used Galerkin and collocation finite element methods for solving fractional diffusion and diffusion-wave equations in the Caputo form. Mohebbi et al. [12] have solved the time fractional nonlinear gas equation arising in quantum mechanics by using of a meshless technique based on collocation and radial basis functions. Hosseini et al. [13] have obtained numerical solution of fractional telegraph equation by applying radial basis functions. Wei et. al. [14] have used fully discrete local discontinuous Galerkin method to solve the fractional telegraph equation.

In this paper, we will use finite element collocation method to obtain the numerical solutions of the time fractional gas dynamics equation with the usage of the $L 1$ discretizaton formula for the fractional derivative as used by Ref. [15]. The equations of gas dynamics are mathematical expressions based on the physical laws of conservation namely, the laws of conservation of mass, conservation of momentum, conservation of energy etc [16]. The various forms of gas dynamics equations have been solved by many authors using different methods and techniques. Among others, Liu [17] has taken some partial differential equations related to gas dynamics and mechanics into consideration and solved them numerically and Rasulov and Karaguler [18] have applied difference scheme to solve some gas dynamics problems.

In the present study, we will take the homogenous nonlinear time fractional gas dynamics equation into consideration as a model given as follows

$$
\frac{\partial^{\gamma} U}{\partial t^{\gamma}}+U \frac{\partial U}{\partial x}-U(1-U)=0
$$

where

$$
\frac{\partial^{\gamma} f(t)}{\partial t^{\gamma}}=\frac{1}{\Gamma(1-\gamma)} \int_{0}^{t}(t-\tau)^{-\gamma} f^{\prime}(\tau) d \tau \quad 0<\gamma \leq 1
$$


is the fractional derivative given in the Caputo's sense $[6,19]$. In the present paper, for the time fractional gas dynamics equation, we will take the boundary conditions of the model problem (1) given in the interval $0 \leq x \leq 1$ as

$$
U(0, t)=E_{\gamma}\left(t^{\gamma}\right), \quad U(1, t)=e^{-1} E_{\gamma}\left(t^{\gamma}\right)
$$

and the initial condition as

$$
U(x, 0)=e^{-x}
$$

The exact solution of the problem is given as [16]

$$
U(x, t)=e^{-x} E_{\gamma}\left(t^{\gamma}\right)
$$

where $E_{\gamma}$ is the Mittag-Leffler function [19].

In our numerical computations, in order to obtain a finite element scheme for solving the fractional gas dynamics equation as Ref. [15] used in explicit finite difference method, we are also going to discretize the Caputo derivative by means of the so-called $L 1$ formula [1]

$$
\left.\frac{\partial^{\gamma} f(t)}{\partial t^{\gamma}}\right|_{t_{m}}=\frac{(\Delta t)^{-\gamma}}{\Gamma(2-\gamma)} \sum_{k=0}^{m-1} b_{k}^{\gamma}\left[f\left(t_{m-k}\right)-f\left(t_{m-1-k}\right)\right]
$$

where

$$
b_{k}^{\gamma}=(k+1)^{1-\gamma}-k^{1-\gamma} .
$$

\section{Cubic B-spline Finite Element Collocation Solutions}

Firstly, we are going to define the cubic B-spline base functions for using in the solution procedure of Eq. (1) with the boundary conditions (2) and the initial condition (3). Regarding that the interval $[a, b]$ is partitioned into $N$ finite elements which have equal length with the knots $x_{m}, m=0,1,2, \ldots, N$ such that $a=x_{0}<x_{1} \cdots<x_{N}=b$ and $h=x_{m+1}-x_{m}$. In [20], the cubic B-splines $\varphi_{m}(x),(m=-1(1) N+1)$, at the knots $x_{m}$ are described over the interval $[a, b]$ as

$$
\varphi_{m}(x)=\frac{1}{h^{3}} \begin{cases}\left(x-x_{m-2}\right)^{3}, & x \in\left[x_{m-2}, x_{m-1}\right], \\ h^{3}+3 h^{2}\left(x-x_{m-1}\right)+3 h\left(x-x_{m-1}\right)^{2}-3\left(x-x_{m-1}\right)^{3}, & x \in\left[x_{m-1}, x_{m}\right], \\ h^{3}+3 h^{2}\left(x_{m+1}-x\right)+3 h\left(x_{m+1}-x\right)^{2}-3\left(x_{m+1}-x\right)^{3}, & x \in\left[x_{m}, x_{m+1}\right], \\ \left(x_{m+2}-x\right)^{3}, & x \in\left[x_{m+1}, x_{m+2}\right], \\ 0 & \text { otherwise. }\end{cases}
$$

A basis is generated for the functions defined over $[a, b]$ by the set of B-splines

$$
\left\{\varphi_{-1}(x), \varphi_{0}(x), \ldots, \varphi_{N+1}(x)\right\} .
$$

Thus, we can write an approximation solution $U_{N}(x, t)$ in terms of the cubic B-splines trial functions as:

$$
U_{N}(x, t)=\sum_{m=-1}^{N+1} \delta_{m}(t) \varphi_{m}(x)
$$


where $\delta_{m}(t)$ 's are unknown, time dependent quantities to be determined from the initial, boundary and cubic B-spline collocation conditions. Since a cubic B-spline covers four consecutive elements, conversely a typical element $\left[x_{m}, x_{m+1}\right]$ is covered by four cubic B-splines. During the solution process of this problem, the finite elements are identified with the interval $\left[x_{m}, x_{m+1}\right]$ and the elements knots $x_{m}, x_{m+1}$. Using the nodal values $U_{m}$ and $U_{m}^{\prime}$ given in terms of the parameter $\delta_{m}(t)$

$$
\begin{aligned}
& U_{m}=U\left(x_{m}, t\right)=\delta_{m-1}(t)+4 \delta_{m}(t)+\delta_{m+1}(t), \\
& U_{m}^{\prime}=U^{\prime}\left(x_{m}, t\right)=\frac{3}{h}\left(-\delta_{m-1}(t)+\delta_{m+1}(t)\right)
\end{aligned}
$$

the variation of $U_{N}(x, t)$ over the typical element $\left[x_{m}, x_{m+1}\right]$ is given by

$$
U_{N}(x, t)=\sum_{j=m-1}^{m+2} \delta_{j}(t) \varphi_{j}(x)
$$

If we replace the global approximation (5) and its required derivatives (6) into Eq. (1), and take $U=z_{m}$, we easily obtain the following set of the $\gamma^{\text {th }}$ order fractional differential equations:

$\dot{\delta}_{m-1}(t)+4 \dot{\delta}_{m}(t)+\dot{\delta}_{m+1}(t)+\frac{3 z_{m}}{h}\left(\delta_{m+1}(t)-\delta_{m-1}(t)\right)+\left(z_{m}-1\right)\left(\delta_{m-1}(t)+4 \delta_{m}(t)+\delta_{m+1}(t)\right)=0$

where "' "denotes $\gamma^{\text {th }}$ fractional derivative with respect to time. If time parameters $\delta_{m}(t)$ 's and its fractional time derivatives $\dot{\delta}_{m}(t)$ 's in Eq. (7) are discretized by the Crank-Nicolson formula and $L 1$ formula, respectively:

$$
\delta=\frac{1}{2}\left(\delta^{n}+\delta^{n+1}\right)
$$

and

$$
\dot{\delta}=\frac{d^{\gamma} \delta}{d t^{\gamma}}=\frac{(\Delta t)^{-\gamma}}{\Gamma(2-\gamma)} \sum_{k=0}^{n-1}\left[(k+1)^{1-\gamma}-k^{1-\gamma}\right]\left[\delta^{n-k}-\delta^{n-k-1}\right],
$$

we obtain a recurrence relationship between successive time levels relating unknown parameters $\delta_{m}^{n+1}(t)$

$$
\begin{aligned}
& \left(1-3 \alpha z_{m}+\alpha h\left(z_{m}-1\right)\right) \delta_{m-1}^{n+1}+\left(4+4 \alpha h\left(z_{m}-1\right)\right) \delta_{m}^{n+1}+\left(1+3 \alpha z_{m}+h \alpha\left(z_{m}-1\right)\right) \delta_{m}^{n+1} \\
& =\left(1+3 \alpha z_{m}-\alpha h\left(z_{m}-1\right)\right) \delta_{m-1}^{n+1}+\left(4-4 \alpha h\left(z_{m}-1\right)\right) \delta_{m}^{n}+\left(1-3 \alpha z_{m}-\alpha h\left(z_{m}-1\right)\right) \delta_{m+1}^{n} \\
& -\sum_{k=1}^{n}\left[(k+1)^{1-\gamma}-k^{1-\gamma}\right]\left[\left(\delta_{m-1}^{n-k+1}-\delta_{m-1}^{n-k}\right)+4\left(\delta_{m}^{n-k+1}-\delta_{m}^{n-k}\right)+\left(\delta_{m+1}^{n-k+1}-\delta_{m+1}^{n-k}\right)\right]
\end{aligned}
$$

where

$$
z_{m}=\delta_{m-1}(t)+4 \delta_{m}(t)+\delta_{m+1}(t)
$$

and

$$
\alpha=\frac{(\Delta t)^{\gamma} \Gamma(2-\gamma)}{2 h} .
$$


The newly obtained iterative system (8) is composed of $N+1$ linear equations including $N+3$ unknown parameters $\left(\delta_{-1}, \ldots, \delta_{N+1}\right)^{T}$. To obtain a unique solution for this system, we are in need of finding two additional constraints. These are obtained from the boundary conditions and their second derivatives and in turn they are used to remove $\delta_{-1}$ and $\delta_{N+1}$ from the system (8) as follows:

$$
\begin{aligned}
& \delta_{-1}(t)=-4 \delta_{0}(t)-\delta_{1}(t)+U\left(x_{0}, t\right) \\
& \delta_{N+1}(t)=-4 \delta_{N}(t)-\delta_{N-1}(t)+U\left(x_{N}, t\right) .
\end{aligned}
$$

Then, this system of equations becomes a matrix equation with $N+1$ unknowns $\mathbf{d}=$ $\left(\delta_{0}, \delta_{1}, \delta_{2}, \ldots, \delta_{N-2}, \delta_{N-1}, \delta_{N}\right)^{T}$ in the form

$$
\mathbf{A d}^{n+1}=\mathbf{B d}^{n} .
$$

\subsection{Initial state}

The initial vector $\mathbf{d}^{0}=\left(\delta_{0}, \delta_{1}, \delta_{2}, \ldots, \delta_{N-2}, \delta_{N-1}, \delta_{N}\right)^{T}$ is determined from the initial and boundary conditions. Now, we have the right to write the approximation (5) for the initial condition as

$$
U_{N}(x, 0)=\sum_{m=-1}^{N+1} \delta_{m}(0) \varphi_{m}(x)
$$

where the $\delta_{m}(0)$ 's are unknown parameters. We require the initial numerical approximation $U_{N}(x, 0)$ satisfy the following conditions:

$$
\begin{array}{ccc}
U_{N}(x, 0) & =U\left(x_{m}, 0\right), & m=0,1, \ldots, N \\
\left(U_{N}\right)_{x x}(0,0) & =1, & \left(U_{N}\right)_{x x}(1,0)=e^{-1} .
\end{array}
$$

Thus, using the these conditions leads to a three-diagonal system of matrix of the form

$$
W \mathbf{d}^{0}=\mathbf{b}
$$

where

$$
W=\left[\begin{array}{cccccccc}
6 & 0 & & & & & & \\
1 & 4 & 1 & & & & & \\
& 1 & 4 & 1 & & & & \\
& & & & \ddots & & & \\
& & & & & 1 & 4 & 1 \\
& & & & & & 0 & 6
\end{array}\right]
$$

and

$$
\mathbf{b}=\left(U\left(x_{0}, 0\right)-\frac{h^{2}}{6} U^{\prime \prime}\left(x_{0}, 0\right), U\left(x_{1}, 0\right), U\left(x_{2}, 0\right), \ldots, U\left(x_{N-2}, 0\right), U\left(x_{N-1}, 0\right), U\left(x_{N}, 0\right)-\frac{h^{2}}{6} U^{\prime \prime}\left(x_{N}, 0\right)\right)^{T} .
$$

\section{$3 \quad$ Numerical examples and results}

In table 1, we compare the analytical solutions with numerical solutions which are obtained by collocation method using cubic B-spline base functions for the time fractional gas dynamics equation. In this table comparisons are mode for the values of $\gamma=0.25, \gamma=0.50$ and $\gamma=0.75$ for $\Delta t=0.0001, t_{f}=0.1$ and $N=40$. As it is seen from the table, the 
analytical and numerical solutions are compatible with each other. Obviously, it is seen that while the values of $\gamma$ increases, the numerical results become more accurate. We can clearly see this situation from the decreasing values of the error norms $L_{2}$ and $L_{\infty}$. Also, in table 2-4, we compare the analytical solutions and numerical solutions for values of $\gamma=0.25, \gamma=0.50$ and $\gamma=0.75$ for $N=80, t_{f}=1$ and different values of $\Delta t$. Obviously, it is seen that from the tables analytical and numerical solutions are compatible with each other. The tables show us that while the number of time steps decreases, the numerical results are more certain. We can clearly see this situation from the decreasing values of the error norms $L_{2}$ and $L_{\infty}$. The error norms $L_{2}$ and $L_{\infty}$ of the analytical solutions and numerical solutions are given for different values of $\gamma$ for $N=80, \Delta t=0.0005$ and different values of $t_{f}$ in Table 5 . In figure 1 , we give the graphs of the analytical solutions and the numerical results for $N=40$ and $\Delta t=0.0001$ at $t=0.1$ (stars), $t=0.5$ (squares), and $t=1.0$ (triangles) for different values of $\gamma$. Consequently, we give five tables to compare our numerical solutions with exact ones. From the figures, it can be said that the numerical and analytical solutions are in good agreement with each other. Additionally acceptable value of error norms $L_{2}$ and $L_{\infty}$ are obtained.

TABLE 1. Comparison of exact solutions with numerical solutions of the problem with $N=40, \Delta t=0.0001$ and $t_{f}=0.1$ at difference $\gamma$

\begin{tabular}{|c|c|c|c|c|c|c|}
\hline \multirow{2}{*}{$x$} & \multicolumn{2}{|c|}{$\gamma=0.25$} & \multicolumn{2}{|c|}{$\gamma=0.50$} & \multicolumn{2}{|c|}{$\gamma=0.75$} \\
\hline & Numerical & Exact & Numerical & Exact & Numerical & Exact \\
\hline 0.0 & 2.364040 & 2.364040 & 1.486763 & 1.486763 & 1.219661 & 1.219661 \\
\hline 0.1 & 2.139072 & 2.139072 & 1.345280 & 1.345279 & 1.103584 & 1.103595 \\
\hline 0.2 & 1.935512 & 1.935512 & 1.217258 & 1.217259 & 0.998541 & 0.998574 \\
\hline 0.3 & 1.751323 & 1.751324 & 1.101416 & 1.101421 & 0.903486 & 0.903547 \\
\hline 0.4 & 1.584663 & 1.584663 & 0.996596 & 0.996607 & 0.817496 & 0.817563 \\
\hline 0.5 & 1.433862 & 1.433863 & 0.901749 & 0.901768 & 0.739704 & 0.739762 \\
\hline 0.6 & 1.297411 & 1.297412 & 0.815929 & 0.815953 & 0.669318 & 0.669364 \\
\hline 0.7 & 1.173946 & 1.173947 & 0.738280 & 0.738305 & 0.605633 & 0.605666 \\
\hline 0.8 & 1.062231 & 1.062232 & 0.668024 & 0.668046 & 0.548009 & 0.548029 \\
\hline 0.9 & 0.961146 & 0.961147 & 0.604460 & 0.604473 & 0.495868 & 0.495877 \\
\hline 1.0 & 0.869682 & 0.869682 & 0.546950 & 0.546950 & 0.448688 & 0.448688 \\
\hline$L_{2} \times 10^{3}$ & 0.195059 & & 0.106440 & & 0.052890 & \\
\hline$L_{\infty} \times 10^{3}$ & 0.317027 & & 0.193832 & & 0.077110 & \\
\hline
\end{tabular}


TABLE 2. The comparison of the exact solutions with the numerical solutions for $\gamma=0.25$, $N=80, t_{f}=1$ and different values of $\Delta t$ and the error norms $L_{2}$ and $L_{\infty}$.

\begin{tabular}{clllll}
\hline$x$ & $\Delta t=0.01$ & $\Delta t=0.005$ & $\Delta t=0.001$ & $\Delta t=0.0005$ & Exact \\
\hline 0.0 & 9.554107 & 9.554107 & 9.554107 & 9.554107 & 9.554107 \\
0.1 & 8.650105 & 8.64671 & 8.644811 & 8.644914 & 8.644914 \\
0.2 & 7.824748 & 7.823211 & 7.822400 & 7.822211 & 7.822242 \\
0.3 & 7.076592 & 7.077950 & 7.077825 & 7.077824 & 7.077857 \\
0.4 & 6.405434 & 6.402297 & 6.404204 & 6.404282 & 6.404310 \\
0.5 & 5.792316 & 5.794983 & 5.794759 & 5.794835 & 5.794859 \\
0.6 & 5.242689 & 5.244285 & 5.243373 & 5.243388 & 5.243405 \\
0.7 & 4.745507 & 4.744555 & 4.744420 & 4.744421 & 4.744429 \\
0.8 & 4.290192 & 4.292500 & 4.292966 & 4.292933 & 4.292937 \\
0.9 & 3.885467 & 3.884501 & 3.884421 & 3.884408 & 3.884410 \\
1.0 & 3.514760 & 3.514760 & 3.514760 & 3.514760 & 3.514760 \\
\hline$L_{2} \times 10^{3}$ & 7.193496 & 3.498592 & 0.685024 & 0.340624 & \\
$L_{\infty} \times 10^{3}$ & 12.962180 & 6.218724 & 1.129466 & 0.548559 & \\
\hline
\end{tabular}

TABLE 3. The comparison of the exact solutions with the numerical solutions for $\gamma=0.50$, $N=80, t_{f}=1$ and different values of $\Delta t$ and the error norms $L_{2}$ and $L_{\infty}$.

\begin{tabular}{clllll}
\hline$x$ & $\Delta t=0.01$ & $\Delta t=0.005$ & $\Delta t=0.001$ & $\Delta t=0.0005$ & Exact \\
\hline 0.0 & 5.008980 & 5.008980 & 5.008980 & 5.008980 & 5.008980 \\
0.1 & 4.531930 & 4.532375 & 4.532313 & 4.532313 & 4.532313 \\
0.2 & 4.101065 & 4.100949 & 4.101007 & 4.101006 & 4.101006 \\
0.3 & 3.710578 & 3.710727 & 3.710745 & 3.710745 & 3.710744 \\
0.4 & 3.357845 & 3.357644 & 3.357623 & 3.357621 & 3.357620 \\
0.5 & 3.038187 & 3.038128 & 3.038104 & 3.038102 & 3.038100 \\
0.6 & 2.749023 & 2.749013 & 2.748991 & 2.748989 & 2.748987 \\
0.7 & 2.487359 & 2.487411 & 2.487391 & 2.487388 & 2.487386 \\
0.8 & 2.250710 & 2.250701 & 2.250684 & 2.250682 & 2.250680 \\
0.9 & 2.036550 & 2.036513 & 2.036502 & 2.036501 & 2.036499 \\
1.0 & 1.842701 & 1.842701 & 1.842701 & 1.842701 & 1.842701 \\
\hline$L_{2} \times 10^{3}$ & 7.407662 & 3.743424 & 0.760637 & 0.381826 & \\
$L_{\infty} \times 10^{3}$ & 11.781515 & 5.958210 & 1.210046 & 0.607343 & \\
\hline
\end{tabular}


TABLE 4. The comparison of the exact solutions with the numerical solutions for $\gamma=0.75$, $N=80, t_{f}=1$ and different values of $\Delta t$ and the error norms $L_{2}$ and $L_{\infty}$.

\begin{tabular}{clllll}
\hline$x$ & $\Delta t=0.01$ & $\Delta t=0.005$ & $\Delta t=0.001$ & $\Delta t=0.0005$ & Exact \\
\hline 0.0 & 3.485866 & 3.485866 & 3.485866 & 3.485866 & 3.485866 \\
0.1 & 3.154202 & 3.154167 & 3.154148 & 3.154145 & 3.154142 \\
0.2 & 2.854084 & 2.854034 & 2.853996 & 2.853991 & 2.853986 \\
0.3 & 2.582519 & 2.582460 & 2.582408 & 2.582401 & 2.582393 \\
0.4 & 2.336811 & 2.336728 & 2.336664 & 2.336655 & 2.336646 \\
0.5 & 2.114462 & 2.114375 & 2.114304 & 2.114295 & 2.114285 \\
0.6 & 1.913265 & 1.913176 & 1.913104 & 1.913094 & 1.913084 \\
0.7 & 1.731196 & 1.731115 & 1.731048 & 1.731039 & 1.731030 \\
0.8 & 1.566432 & 1.566368 & 1.566315 & 1.566308 & 1.566301 \\
0.9 & 1.417323 & 1.417286 & 1.417256 & 1.417252 & 1.417247 \\
1.0 & 1.282379 & 1.282379 & 1.282379 & 1.282379 & 1.282379 \\
\hline$L_{2} \times 10^{3}$ & 6.545792 & 3.420635 & 0.737417 & 0.377396 & \\
$L_{\infty} \times 10^{3}$ & 10.356218 & 5.407458 & 1.164772 & 0.595996 & \\
\hline
\end{tabular}

TABLE 5. The numerical solutions with $N=80$ and $\Delta t=0.0005$ for different values of $t_{f}$ and the error norms $L_{2}$ and $L_{\infty}$.

\begin{tabular}{|c|c|c|c|c|c|c|}
\hline \multirow[b]{2}{*}{$t_{f}$} & \multicolumn{2}{|c|}{$\gamma=0.25$} & \multicolumn{2}{|c|}{$\gamma=0.50$} & \multicolumn{2}{|c|}{$\gamma=0.75$} \\
\hline & $L_{2} \times 10^{3}$ & $L_{\infty} \times 10^{3}$ & $L_{2} \times 10^{3}$ & $L_{\infty} \times 10^{3}$ & $L_{2} \times 10^{3}$ & $L_{\infty} \times 10^{3}$ \\
\hline 0.2 & 0.699898 & 1.127687 & 0.502329 & 0.870746 & 0.256093 & 0.447387 \\
\hline 0.4 & 0.500072 & 0.803301 & 0.467573 & 0.755646 & 0.312253 & 0.590927 \\
\hline 0.6 & 0.415936 & 0.667972 & 0.438989 & 0.700142 & 0.359862 & 0.581380 \\
\hline 0.8 & 0.369532 & 0.594400 & 0.409807 & 0.651525 & 0.369240 & 0.578283 \\
\hline
\end{tabular}



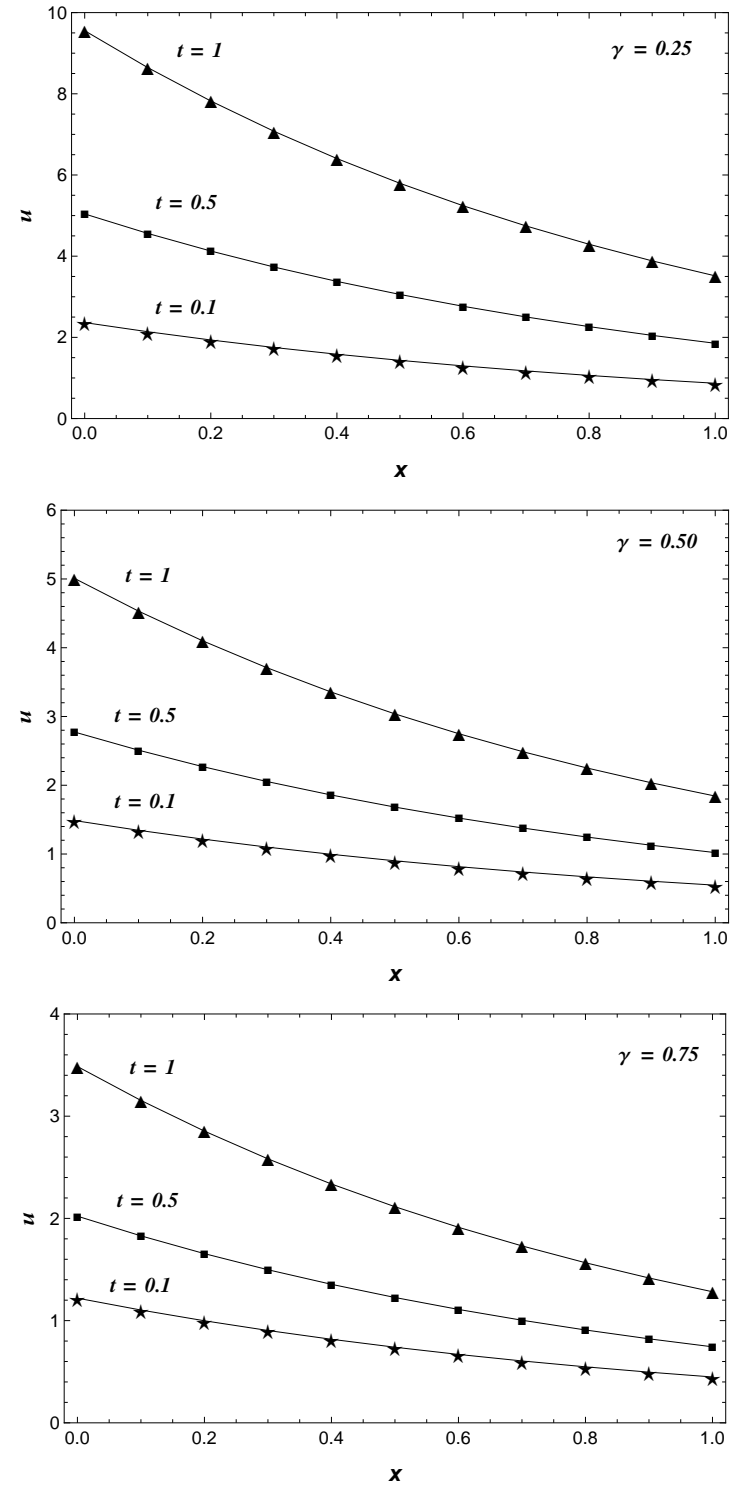

FiguRE 1. The comparison of the exact(lines) and numerical solutions for different values of $\gamma, N=40$ and $\Delta t=0.0001$ at $t=0.1$ (stars), $t=0.5$ (squares), and $t=1.0$ (triangles). 


\section{Conclusion}

In the present study, a finite element collocation method has been successfully applied to obtain the numerical solutions of the time fractional gas dynamics equation. In the equation, the time fractional derivative has been taken into consideration in the form of the Caputo sense. The fractional derivative appearing in the time fractional gas equation has been approximated by means of the so-called $L 1$ formulae. One can easily conclude from the presented results that the applied method is a highly good one to obtain numerical solutions of this kind fractional partial differential equations. The easy application and programming of the method put it in a better position with respect to other methods. Moreover, it can be used for a different problem with little manipulations on the scheme. In conclusion, it can be said that the present method can also be used for a wide range of fractional problems existing in various fields of science.

\section{References}

[1] K. B. Oldham and J. Spanier, The fractional calculus, Academic, New York, 1974.

[2] J. Singh, D. Kumar and A. Kilicman, Homotopy perturbation method for fractional gas dynamics equation using Sumudu transform, Abstr. Appl. Anal. (2013), http://dx.doi.org/10.1155/2013/934060, Article ID 934060, 8 pp.

[3] L. Debnath, Fractional integral and fractional differential equations in fluid mechanics, Fract. Calc. Appl. Anal. 6 (2003) 119-155.

[4] S. Monami and Z. Odibat, Analytical approach to linear fractional partial differential equations arising in fluid mechanics, Phys. Lett. A 355 (2006) 271-279.

[5] D.L. Logan, A first course in the finite element method (Fourth Edition), Thomson, 2007.

[6] A. A. Kilbas, H.M. Srivastava and J.J. Trujillo, Theory and applications of fractional differential equations, Elsevier, Amsterdam, 2006.

[7] S. S. Ray, Exact solutions for time-fractional diffusion-wave equations by decomposition method, Phys. Scr. 75 (2007) 53-61.

[8] O.P. Agrawal, Solution for a fractional diffusion-wave equation defined in a bounded domain, Nonlin. Dynam. 29 (2002) 145-155.

[9] H. Jafari and S.Momani, Solving fractional diffusion and waves equations by modifiying homotopy perturbation method, Phys. Lett. A 370 (2007) 388-396.

[10] A. Esen, Y. Ucar, N. Yagmurlu and O. Tasbozan, A Galerkin finite element method to solve fractional diffusion and fractional diffusion-wave equations, Math. Model. and Anal. 18 (2013) 260-273. 
[11] A. Esen, O. Tasbozan, Y. Ucar and N.M. Yagmurlu, A B-spline collocation method for solving fractional diffusion and fractional diffusion-wave equations, Tbilisi Mathematical Journal 8 (2015) 181-193.

[12] A. Mohebbi, A. Mostafa and M. Dehghan, The use of a meshless technique based on collocation and radial basis functions for solving the time fractional nonlinear Schrödinger equation arising in quantum mechanics, Eng. Anal. with Bound. Elem. 37 (2013) 475-485.

[13] V. R. Hosseini, W. Chen and Z. Avazzade, Numerical solution of fractional telegraph equation by using radial basis functions, Eng. Anal. with Bound. Elem. 38 (2014) 31-39.

[14] L. Wei, H. Dai, D. Zhang and Z. Si, Fully discrete local discontinuous Galerkin method for solving the fractional telegraph equation, Calcolo 51 (2014) 175-192.

[15] J. Q. Murillo and S.B. Yuste, An explicit difference method for solving fractional diffusion and diffusion-wave equations in the Caputo form, J. Comput. Nonlinear Dynam. 6 (2011) 021014.

[16] S. Das and R. Kumar, Approximate analytical solutions of fractional gas dynamic equations, Appl. Math. and Comput. 217 (2011) 9905-9915.

[17] T-P. Liu, Nonlinear waves in mechanics and gas dynamics, Defense Technical Information Center Accession Number: ADA 238340 (1990).

[18] M. Rasulov and T. Karaguler, Finite difference schemes for solving system equations of gas dynamic ina class of discontinuous functions, Appl. Math. and Comput. 143 (2003) 45-164.

[19] I. Podlubny, Fractional differential dquations, Academic Press, San Diego, 1999.

[20] P. M. Prenter, Splines and variasyonel methods, New York, John Wiley, 1975. 